\title{
Hindlimb Suspension Does Not Influence Mechanical Sensitivity, Epidermal Thickness, and Peripheral Nerve Density in the Glabrous Skin of the Rat Hind Paw
}

\author{
Y. TANAKA ${ }^{1}$, J. NAKANO ${ }^{2}$, Y. HAMAUE ${ }^{3}$, Y. SEKINO ${ }^{3}$, J. SAKAMOTO ${ }^{4}$, H. KATAOKA ${ }^{3}$, \\ M. OKITA ${ }^{3}$
}

${ }^{1}$ Department of Rehabilitation, Nagasaki Memorial Hospital, Nagasaki-shi, Japan, ${ }^{2}$ Unit of Physical Therapy and Occupational Therapy Sciences, Nagasaki University Graduate School of Biochemical Sciences, Nagasaki-shi, Japan, ${ }^{3}$ Department of Locomotive Rehabilitation Science, Unit of Rehabilitation Sciences, Nagasaki University Graduate School of Biomedical Sciences, Nagasakishi, Japan, ${ }^{4}$ Department of Rehabilitation, Nagasaki University Hospital, Nagasaki-shi, Japan

Received March 6, 2012

Accepted September 24, 2012

On-line November 22, 2012

\section{Summary}

Our aim was to investigate the influence of microgravity on the sensitivity of the skin to mechanical stimulation, epidermal thickness, peripheral nerve density in the upper dermis, and serum levels of a stress marker in a rat hindlimb suspension (HS) model. Thirty 8-week-old male Wistar rats were randomly divided into 3 groups: HS, $n=10$; sham $H S, n=10$; control, $n=10$. The suspension system was attached to rat tails in both the HS and sham-HS groups, but the hindlimbs were suspended only in the HS group. The HS and sham-HS groups were treated for 4 weeks. In behavioral tests using von-Frey filaments $(n=5$ in each group), mechanical hypersensitivity developed in the HS and sham HS groups. Serum corticosterone levels increased significantly in the HS and sham HS groups compared to the control group, and no changes in epidermal thickness or peripheral nerve density were observed immediately after the removal of HS ( $n=5$ in each group). These data indicated that the mechanical hypersensitivity observed in the HS group was not caused by microgravity or inactivity, but rather by restraint stress. We suggest that microgravity does not affect skin sensitivity and histology in these animals.

\section{Key words}

Hindlimb suspension - Mechanical hypersensitivity • Epidermal thickness $\bullet$ Peripheral nerve $\bullet$ Stress

\section{Corresponding author}

Jiro Nakano, Unit of Physical Therapy and Occupational Therapy Sciences, Nagasaki University Graduate School of Biochemical Sciences, 1-7-1 Sakamoto, Nagasaki-shi 852-8520, Japan. Fax: +81-95-819-7919. E-mail: nakano-j@nagasaki-u.ac.jp

Hindlimb suspension (HS) animal models are widely used in studies investigating the effect of microgravity and spaceflight on the locomotorium such as bone and muscle (Templeton et al. 1984). Changes in the viscera (Stein et al. 2005), circulation (De Salvatore et al. 2004), and blood vessels (Fujino et al. 2005) have also been studied using the HS model. A few histochemical studies that investigated muscular sensation in response to HS showed no effect on muscle spindles or the size and shape of Golgi tendon organs (Asmussen and Soukup 1991). However, extremely few studies have addressed the changes in skin tissues and peripheral nerves or the sensory aspects of suspension hypokinesia. Changes in skin tissue and peripheral nerves may affect the skin sensation (Li et al. 2009, Nakano et al. 2012). Although a few reports showed that microgravity and non-weight bearing are associated with pressure hypersensitivity in rats, the effect of stress was not examined using a sham group in these studies 
(Chowdhury et al. 2011, Kauppila et al. 1998). It is well known that various stresses can induce hypersensitivity (Bardin et al. 2009, Imbe et al. 2004, Nasu et al. 2009). In studies using HS model rats, the influence of restraint stress should not be ignored because HS affects the wellness of the animal. A previous study showed increased serum levels of stress markers such as epinephrine in HS model rats (Kawano et al. 1995). Thus, whether hypersensitivity is a specific consequence of microgravity, or if other stimuli can also cause it, remains unclear. To address these issues, we examined the changes in the sensitivity of the skin in response to mechanical stimuli, epidermal thickness, peripheral nerve density in the upper dermis, and serum levels of a stress marker by using HS model rats.

This study was approved by the Ethics Review Committee for Animal Experimentation of Nagasaki University. Thirty 8-week-old male Wistar rats weighing $220 \pm 18$ g from Kudo Laboratories (Tokyo, Japan) were randomly divided into control, HS, and sham-HS groups ( $n=10$ each). Rats were housed individually in $30 \times 40 \times$ $20 \mathrm{~cm}$ cages at $24^{\circ} \mathrm{C}$, with water and food available $\mathrm{ad}$ libitum. The suspension system was attached to the tail of rats in the HS group, and the height of the system was adjusted so that the suspended hindlimbs did not touch the floor (Templeton et al. 1984). The same suspension system was attached to the tails of rats in the sham-HS group but the hindlimbs were not suspended, so the rats could move normally in their cages. All other aspects of the set-up in the three groups were identical. The period of HS was 4 weeks.

Behavioral tests to assess bilateral hindlimb mechanical sensitivity were performed weekly in 5 rats from each group. Rats were placed in a small animal fixator in a quiet room during the behavioral test. The glabrous skin of the hind paw was probed 10 times with a von Frey filament (15 g; North Coast Medical, USA) repeatedly, and the paw-withdrawal response (PWR) was determined. The body weights of rats in the 3 groups were also measured weekly. At the end of the HS period, the suspension system was removed in the HS and shamHS groups. The rats that underwent behavioral testing were maintained in a normal breeding state, and the behavioral tests and weight measurements were extended for 8 weeks after the removal of HS. The other 5 rats in each group that were not subjected to behavioral tests were intraperitoneally anesthetized with sodium pentobarbital $(40 \mu \mathrm{g} / \mathrm{g})$ immediately after the removal of $\mathrm{HS}$, and blood was collected by venipuncture of the tail.
The serum was extracted after clotting and centrifugation of blood, and it was stored in the freezer. Subsequently, transcardial perfusion was performed with $4 \%$ paraformaldehyde in $0.1 \mathrm{M}$ phosphate buffer, and the glabrous skin of the hind paw was removed bilaterally. The tissues were embedded in tragacanth gum after soaking in $10 \%$ saccharose and stored at $-80{ }^{\circ} \mathrm{C}$. Serum corticosterone levels were estimated as a stress marker using the Corticosterone EIA kit (YK240; Yanaihara Institute Inc., Japan) (Hong et al. 2011). Although the influence of pentobarbital anesthesia cannot not be ignored in the determination of serum corticosterone levels (Green and Moor 1977), its effect on the results was minimized by employing uniform anesthesia conditions for all rats during blood sampling. Frozen skin sections $(10 \mu \mathrm{m})$ were cut using a cryostat (CM1950; Leica, Germany) and stained with hematoxylin-eosin. Epidermal thickness was measured using the Scion Image software (Scion Corp., USA) at 7 random points per image $(\times 200)$. Five sections per skin sample were used for measurements. Immunohistochemical staining for peripheral nerves was performed on frozen sections $(30 \mu \mathrm{m})$ with rabbit anti-PGP9.5 antibody (1:2000; Calbiochem, Germany), as previously described (Lauria et al. 2005). For semiquantitative analysis, the number of PGP9.5-positive nerve fiber fragments in the upper dermis $(0-50 \mu \mathrm{m}$ in depth under the epidermis) was counted on the images $(\times 200)$ obtained from 5 sections per skin sample and classified according to the skin tissue width. The number of PGP9.5 nerve fibers per $1 \mathrm{~mm}$-unit was calculated as "peripheral nerve profiles." Data are presented as mean \pm SEM. Statistical analysis was performed using ANOVA with the Scheffe post-hoc test, and $\mathrm{P}<0.05$ was considered significant.

Although body weight increased at all time points in the HS, sham-HS, and control groups, weight gain in the HS and sham-HS groups was lower than that in the control group (Fig. 1A). The PWR increased significantly during $\mathrm{HS}$ in the HS and sham-HS groups compared to the control group ( $\mathrm{P}<0.01$ ), and this increase in the PWR persisted for 5 and 6 weeks after removal of HS (Fig. 1B). However, no significant differences in body weight and PWR were observed between the HS and sham-HS groups. Serum corticosterone levels increased significantly in the HS and sham-HS groups compared to the control group $(\mathrm{P}<0.01)$ (Fig. 2A), with no significant differences in corticosterone levels between these 2 groups. In the histological analysis, no pathological changes such as inflammation were 
observed in skin tissue. No significant changes in epidermal thickness or peripheral nerve profiles in the

A

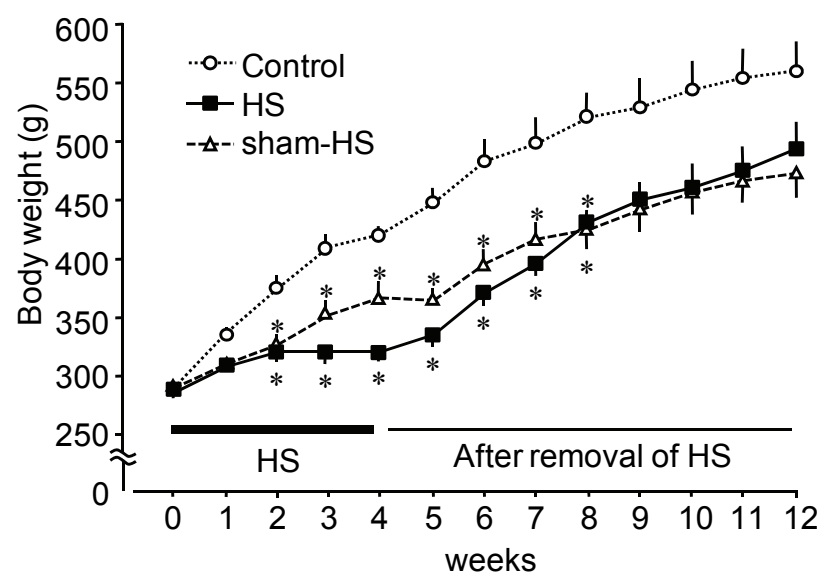

glabrous skin of the hind paw were observed in any of the groups (Fig. 2B,C).

B

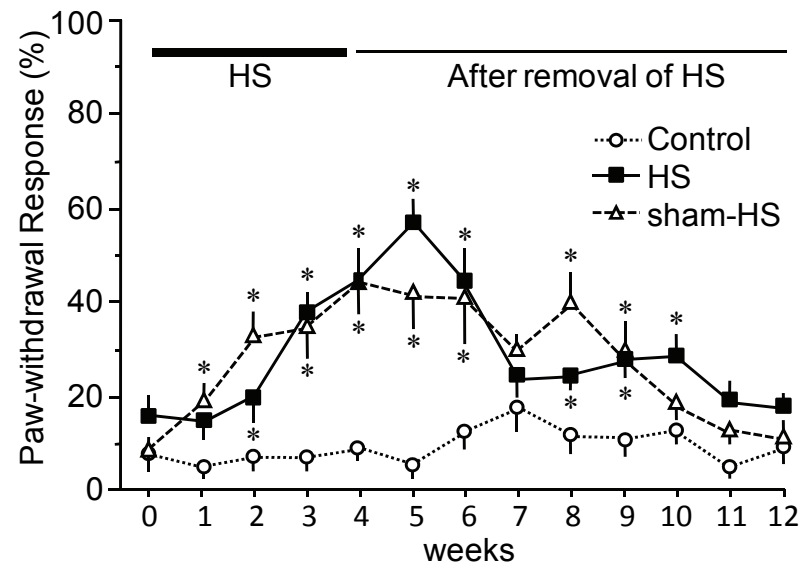

Fig. 1. Changes in body weight (A) and mechanical sensitivity (B) during and after HS. The HS and sham-HS procedures resulted in a significant decrease in weight gain; no significant difference was observed between the HS and sham-HS groups (A). The PWR induced by $15-\mathrm{g}$ von Frey filaments significantly increased in the HS and sham-HS groups during HS compared to the control group; no significant difference was observed between the HS and sham-HS groups (B). HS: hindlimb suspension. * vs. control group, $\mathrm{P}<0.05$.

A

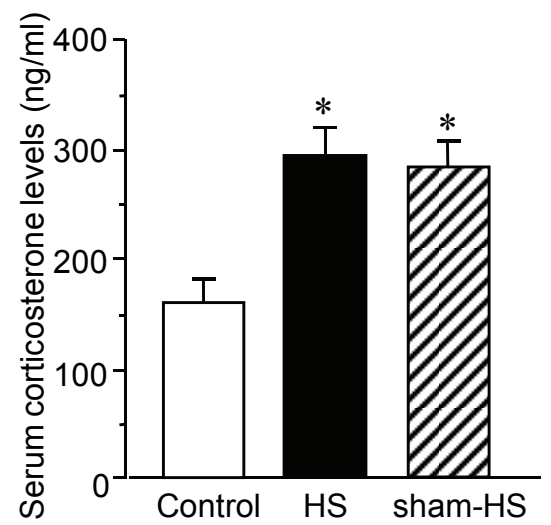

B

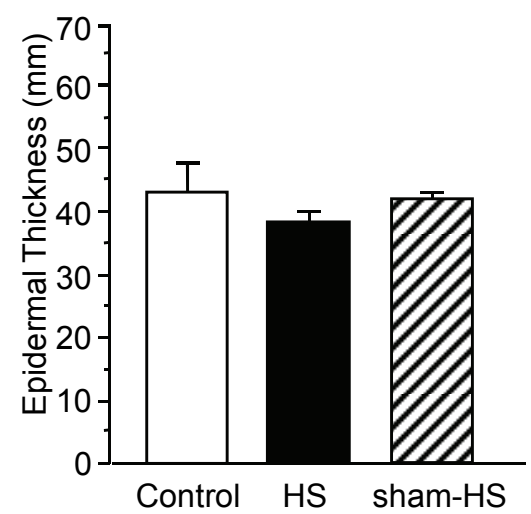

C

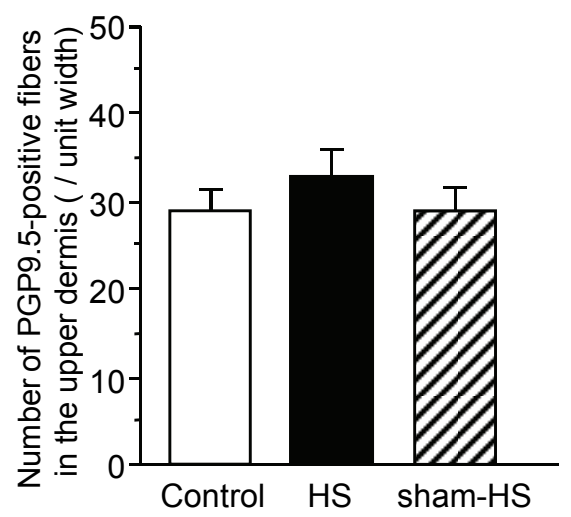

Fig. 2. Changes in serum corticosterone levels (A), epidermal thickness (B), and peripheral nerve density in the upper dermis (C) immediately after the removal of HS. Serum corticosterone levels significantly increased in the HS and sham-HS groups compared to the control group; no significant difference was observed between the HS and sham-HS groups. The procedures performed in the HS and sham-HS groups did not cause changes in epidermal thickness and density of PGP9.5-positive nerve fibers in the upper dermis. * vs. control group, $\mathrm{P}<0.05$.

No differences were observed between the HS and sham-HS groups in any of the parameters tested. Chowdhury et al. (2011) suggested that non-weight bearing conditions in HS model rats may lead to pressure hypersensitivity. In the present study, although the same HS model was used, mechanical hypersensitivity was observed in the sham-HS group as well. Rats in the shamHS group were attached to the trolley suspension system in the same manner as those in the HS group during HS, and they were allowed to bear weight on their hindlimbs while walking. Our results indicate that the mechanical hypersensitivity observed during HS cannot be attributed to the effects of microgravity. In addition, changes in epidermal thickness and peripheral nerve density, which are often observed in diabetes and neuropathy (Chao et al. 2011, Kojundzic et al. 2008, Lauria et al. 2005, Romanovsky et al. 2010), were not detected in the HS group. These results indicate that the hypersensitivity in the HS group was not due to histological changes of peripheral tissues such as skin and nerve. Because a 
similar increase in the serum levels of corticosterone, which was used as a stress marker, was observed in the HS and sham groups, it is possible that mechanical hypersensitivity in these groups was induced by stress. Body weight analysis confirmed the involvement of stress. Attachment of the suspension system may have resulted in the development of restraint stress and possibly mechanical hypersensitivity (Bardin et al. 2009, Imbe et al. 2004, Nasu et al. 2009).

Inactivity and immobilization of extremities, such as those associated with casting after fracture, have been reported to induce hypersensitivity (Allen et al. 1999, Guo et al. 2004). Terkelsen et al. (2008) conducted an experiment involving healthy human subjects in which the forearm was immobilized by casting for 4 weeks; subsequently, transient movement-provoked pain and increased skin temperature in concert with mechanical and cold hyperalgesia were induced in distal parts of the immobilized extremity. Although the detailed mechanisms are unclear, several factors have been associated with the induction of hypersensitivity, including hypokinesia; reduction of the sensory input; changes in peripheral tissue, including the skin; blood flow deficiencies; central neuron system changes; and stress. In our HS model, and considering the results of the study, hypokinesia, reduction of sensory input, and changes in the skin tissue can be excluded. However, stress may have affected the results of various parameters, and further investigation will be necessary in the future.

In conclusion, mechanical hypersensitivity observed in a HS model rat was related to stress but not to microgravity. In addition, our results indicated that microgravity did not affect the sensitivity or histology of the glabrous skin.

\section{Conflict of Interest}

There is no conflict of interest.

\section{Acknowledgements}

This study was supported by a grant-in-aid for scientific research (No. 23500587) from the Japan Society for the Promotion of Science. The authors thank Takuya Fukushima (Department of Rehabilitation, Shimonoseki City Central Hospital, Shimonoseki-shi, Japan) for his assistance in the experimental work.

\section{References}

ALLEN G, GALER BS, SCHWARTZ L: Epidemiology of complex regional pain syndrome: a retrospective chart review of 134 patients. Pain 80: 539-544, 1999.

ASMUSSEN G, SOUKUP T: Arrest of developmental conversion of type II to type I fibres after suspension hypokinesia. Histochem J 23: 312-322, 1991.

BARDIN L, MALFETES N, NEWMAN-TANCREDI A, DEPOORTERE R: Chronic restraint stress induces mechanical and cold allodynia, and enhances inflammatory pain in rat: Relevance to human stress-associated painful pathologies. Behav Brain Res 205: 360-366, 2009.

CHAO CY, ZHENG YP, CHEING GL: Epidermal thickness and biomechanical properties of plantar tissues in diabetic foot. Ultrasound Med Biol 37: 1029-1038, 2011.

CHOWDHURY P, SOULSBY ME, JAYROE J, AKEL NS, GADDY D, DOBRETSOV M: Pressure hyperalgesia in hind limb suspended rats. Aviat Space Environ Med 82: 988-991, 2011.

De SALVATORE G, DESAPHY JF, PIEPOLi AL, NATAlE L, De SALVIA MA, MitOlO CI, RENNA G, CONTECAMERINO D, MITOLO-CHIEPPA D: Functional alterations of mesenteric vascular bed, vas deferens and intestinal tracts in a rat hindlimb unloading model of microgravity. Auton Autacoid Pharmacol 24: 45-54, 2004.

FUJINO H, KOHZUKI H, TAKEDA I, KIYOOKA T, MIYASAKA T, MOHRI S, SHIMIZU J, KAJIYA F: Regression of capillary network in atrophied soleus muscle induced by hindlimb unweighting. J Appl Physiol 98: 1407-1413, 2005.

GREEN D, MOOR RM: The influence of anaesthesia on the concentrations of progesterone and cortisol in peripheral blood plasma of sheep. Res Vet Sci 22: 122-123, 1977.

GUO TZ, OFFLEY SC, BOYD EA, JACOBS CR, KINGERY WS: Substance P signaling contributes to the vascular and nociceptive abnormalities observed in a tibial fracture rat model of complex regional pain syndrome type $\mathrm{I}$. Pain 108: 95-107, 2004. 
HONG S, ZHENG G, WU X, SNIDER NT, OWYANG C, WILEY JW: Corticosterone mediates reciprocal changes in CB 1 and TRPV1 receptors in primary sensory neurons in the chronically stressed rat. Gastroenterology 140 : 627-637, 2011.

IMBE H, MURAKAMI S, OKAMOTO K, IWAI-LIAO Y, SENBA E: The effects of acute and chronic restraint stress on activation of ERK in the rostral ventromedial medulla and locus coeruleus. Pain 112: 361-371, 2004.

KAUPPILA T, KONTINEN VK, PERTOVAARA A: Weight bearing of the limb as a confounding factor in assessment of mechanical allodynia in the rat. Pain 74: 55-59, 1998.

KAWANO S, OHMORI S, KAMBE F, KANDA K, MURATA Y, SEO H: Catecholamine response to stress: age related modifications in tail-suspended rats. Environ Med 39: 107-111, 1995.

KOJUNDZIC SL, DUJMOVIC I, GRKOVIC I, SAPUNAR D: Regional differences in epidermal thickness and behavioral response following partial denervation of the rat paw. Int J Neurosci 118: 1748-1762, 2008.

LAURIA G, LOMBARDI R, BORGNA M, PENZA P, BIANCHI R, SAVINO C, CANTA A, NICOLINI G, MARMIROLI P, CAVALETTI G: Intraepidermal nerve fiber density in rat foot pad: neuropathologicneurophysiologic correlation. J Peripher Nerv Syst 10: 202-208, 2005.

LI WW, SABSOVICH I, GUO TZ, ZHAO R, KINGERY WS, CLARK JD: The role of enhanced cutaneous IL-1beta signaling in a rat tibia fracture model of complex regional pain syndrome. Pain 144: 303-313, 2009.

NAKANO J, SEKINO Y, HAMAUE Y, SAKAMOTO J, YOSHIMURA T, ORIGUCHI T, OKITA M: Changes in hind paw epidermal thickness, peripheral nerve distribution and mechanical sensitivity after immobilization in rats. Physiol Res 61: 643-647, 2012.

NASU T, TAGUCHI T, MIZUMURA K: Persistent deep mechanical hyperalgesia induced by repeated cold stress in rats. Eur J Pain 14: 236-244, 2009.

ROMANOVSKY D, WANG J, AL-CHAER ED, STIMERS JR, DOBRETSOV M: Comparison of metabolic and neuropathy profiles of rats with streptozotocin-induced overt and moderate insulinopenia. Neuroscience 170: 337-347, 2010.

STEIN TP, SCHLUTER MD, GALANTE AT, SOTEROPOULOS P, RAMIREZ M, BIGBEE A, GRINDELAND RE, WADE CE: Effect of hind limb muscle unloading on liver metabolism of rats. J Nutr Biochem 16: 9-16, 2005.

TEMPLETON GH, PADALINO M, MANTON J, GLASBERG M, SILVER CJ, SILVER P, DEMARTINO G, LECONEY T, KLUG G, HAGLER H, ET AL.: Influence of suspension hypokinesia on rat soleus muscle. J Appl Physiol 56: 278-286, 1984.

TERKELSEN AJ, BACH FW, JENSEN TS: Experimental forearm immobilization in humans induces cold and mechanical hyperalgesia. Anesthesiology 109: 297-307, 2008. 